\title{
Methotrexate Overdose
}

\author{
Avinash Aujayeb ${ }^{1^{*}}$ \\ ${ }^{1}$ Consultant in General and Respiratory Medicine, Elysium HealthCare Ltd, Level 3, Alexander House, Cyber City, Mauritius
}

\begin{abstract}
Received: March 30, 2017; Accepted: April 15, 2017; Published: April 20, 2017
\end{abstract}
*Corresponding author: Avinash Aujayeb, Consultant in General and Respiratory Medicine, Elysium HealthCare Ltd, Level 3, Alexander House, Cyber City, Mauritius. E-mail: aujayeb@doctors.org.uk

\begin{abstract}
A healthy male patient was dispensed Methotrexate by mistake. He presented with typical features of toxicity and treating him proved challenging in a resource poor country

Introduction: A male patient took regular high dose Methotrexate by mistake.

Case report: He presented with liver dysfunction, dermatological and haematological problems which were life threatening. Prompt treatment prevented ongoing complications.
\end{abstract}

Discussion: This is centred on how methotrexate acts as well as how to reverse its effects, in a resource poor country.

Conclusion: This is likely to be an unreported problem and it is important that all emergency physicians know how to manage this problem.

Keywords: Methotrexate; Overdose

\section{Introduction}

$\mathrm{KM}$ is male and 34 years of age with no past medical history. He had no recent travel and is married with 2 children. He lives on the island of Mauritius. He presented to a local private dermatologist with a subcutaneous infection on the dorsum of both of his hands, and was prescribed a five-day course of ciprofloxacin 500mg twice a day and metronidazole 500mg tds. He presented the prescription as shown in Figure 1 to a private pharmacy and duly started taking the medication given to him.

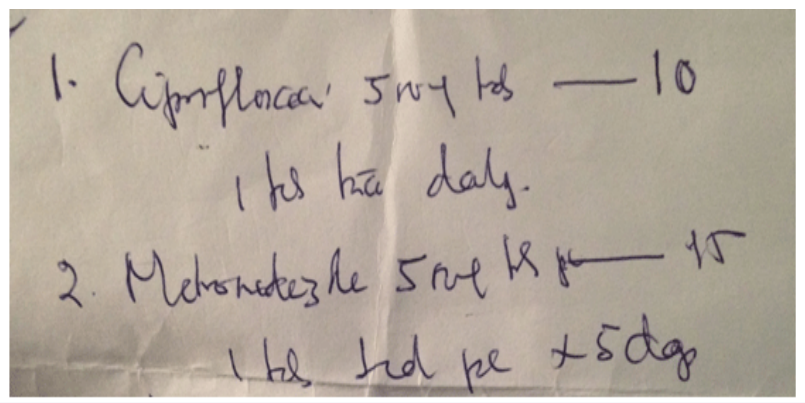

Figure 1: Prescription clearly showing metronidazole and ciprofloxacin
He presented 5 days later to a private clinic with presenting complaints of lethargy, dizziness, nausea, difficulty swallowing, painful and bleeding mouth ulcerations, inability to withstand the sun, an infected left sided wound from falling over and headaches.

It was then noted that he had been taking methotrexate $5 \mathrm{mg}$ tds, and had ingested $75 \mathrm{mg}$ in the last 5 days rather than metronidazole.

\section{Case Report}

On examination, he had widespread angular stomatitis with bleeding oral ulcerations. He had a very faint widespread macular rash over his torso and his hand infection had almost completely disappeared, as shown in Figure 2. His cardiovascular, respiratory, abdominal and neurological examination was normal. He had a $2 \mathrm{~cm}$ by $3 \mathrm{~cm}$ wound on the anterior aspect of his left leg which was oozing minimal pus. He was apyprexial, normotensive and his pulse was 87 beats per minute. He had a BMI of 33 .

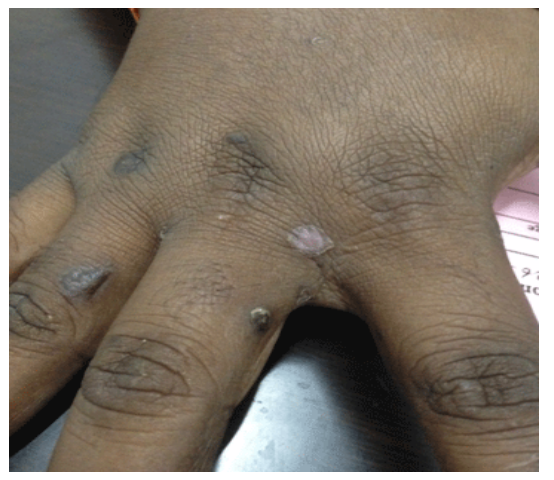

Figure 2: Picture of hand showing healing subcutaneous lesions

He only had one small resolving cutaneous lesion on his left thumb, from the infection that was being treated.

His electrocardiogram was normal and his blood tests showed neutropenia with thrombocytopenia, deranged liver function tests in a hepatitic pattern but a normal coagulation and kidney profile. 
A diagnosis of acute methotrexate (Mtx) toxicity was made. It is not possible to do methotrexate levels on the island.

The patient was isolated and started on broad spectrum intravenous antibiotics, analgesics and mouthwashes. Intravenous fluids were prescribed to keep the urine output to over $100 \mathrm{mls} /$ hour to help flush out any metabolites. The neutropenia and hepatitis were worrisome and it was felt that the patient required intravenous folinic acid to reverse the methotrexate [1]. However, this was unable to be sourced in any of the private or government hospitals at the time of presentation which was quite late at night. There was no Granulocyte-Colony Stimulating Factor (G-CSF or GCSF) available either.

The following day, his liver function tests worsened and his neutropenia persisted. The Chief Pharmacy Officer in the Ministry of Health was contacted and intravenous folinic acid was sourced and bought for the patient. 15mg were given every 6 hours. fatty liver

An abdominal ultrasound also showed that he had a

Over the next 4 days, his oral ulcers and leg wound improved and his various symptoms disappeared.

Figure 3 illustrates the pattern of his blood results. With the folinic acid, his hepatitis improved markedly but showed a slight upward trend and then levelled out which might be due to his fatty liver disease. His platelet and neutrophil count all normalized after 2 weeks.

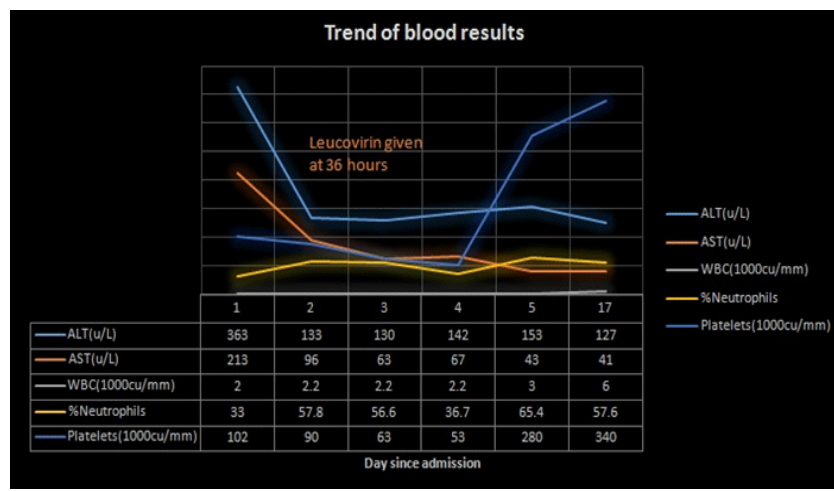

Figure 3: Graph showing serial liver function tests, white cell, platelet and neutrophil counts and variation after administration of leucovorin

However, his hair thinned and fell out over that period as shown in Figure 4. Serum folic acid levels were normal but he continues $5 \mathrm{mg}$ supplementation per day and is under regular follow up.

\section{Discussion}

Methotrexate is a folate analogue and an anti-metabolite that is commonly used in the treatment of psoriasis, rheumatoid arthritis and some forms of cancer. It has some use in asthma as well. It inhibits folic acid synthesis and thus affects DNA synthesis and repair as well as cellular replication. Excretion is predominantly renal, with only a small proportion undergoing

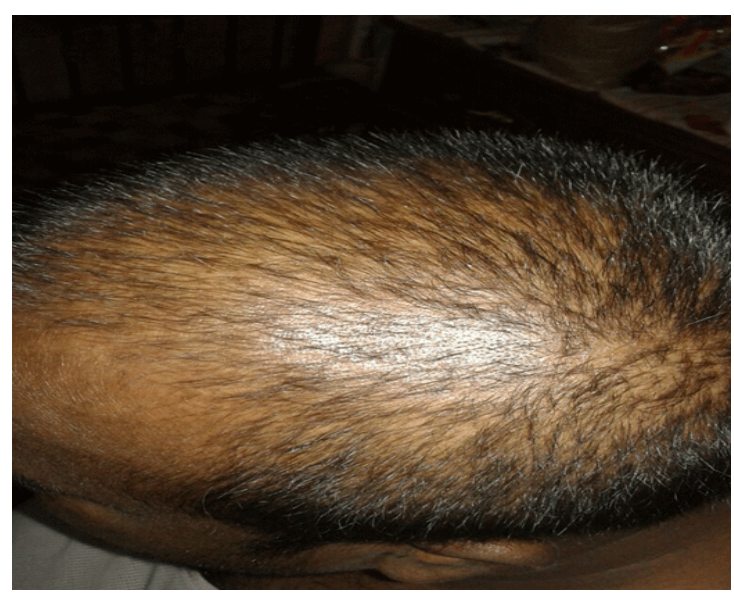

Figure 4: Picture showing hair thinning. This eventually all fell out.

hepatic first pass metabolism [2].

As with many other anticancer drugs, methotrexate has little selectivity for tumor cells, and its effectiveness is limited by toxicity to normal tissue, particularly the Gastro Intestinal (GI) epithelium and bone marrow. By providing reduced folates (leucovorin, also called folinic acid, N5-formyl-tetrahydrofolate, citrovorum factor) to bypass the metabolic block induced by methotrexate, within 24 to 36 hours after administration of methotrexate, normal cells that have not already had lethal DNA damage can be rescued $[3,4]$.

Clinical features of acute methotrexate toxicity are varied1. Neurological symptoms such as drowsiness and confusion can occur. Pulmonary infiltrates are usually associated with chronic use. An acute transaminitis may occur in 60 to 80 percent of patients and typically resolves spontaneously within one to two weeks. Typically, the bilirubin levels remain normal. Nausea, vomiting and stomatitis are very common and avoided if leucovorin is used concurrently in high dose methotrexate therapy. Such doses can also induce direct renal tubular injury but that risk can be reduced by hydration both to maintain a high urine flow to lower the concentration of methotrexate in the tubular fluid and by alkalinisation of the urine to a $\mathrm{pH}$ above 7.0 to increase the solubility of the drug ${ }^{2}$. The latter was not possible in the clinic.

Approximately 14 to 15 percent of patients develop a nonspecific morbilliform drug rash, which is usually erythematous, macular, pruritic, and often confined to the neck and trunk. These rashes can be photosensitive. Myelosuppression and hair loss can also be a transient problem as was the case here.

\section{Conclusion}

The pharmacy implicated in the drug error apologized to the patient and paid the full costs of the inpatient stay.

It was also noted that the metronidazole prescription was not clear in terms of the dosage. Mauritius does not have any policies such as the United Kingdom's duties of a doctor which 
states that all doctors' handwriting should be clear and legible [5]. A memorandum to this effect has actually been circulated to all registered doctors in Mauritius Medical Council. A further improvement would be to implement electronic prescriptions but Mauritius does not have even an electronic system for radiology.

The healthcare system in Mauritius is divided unequally between the private and public sectors, and many resources are not available in the former, such as the above emergency treatment and thus needed some negotiations to source.

There is no established system for reporting errors in Mauritius, and previous experience suggests that any such reports result in punitive action rather than formative ones. The local pharmacy board was made aware of the error but I was told that without a formal complaint from the patient, this could not be actioned. The patient decided not to put a complaint in as the pharmacy paid all the fees of his treatment in the private sector and offered an apology.

Personally, I think this is a troubling matter as anecdotally, there is gross under-reporting of drug errors in Mauritius. As such, this potentially represents significant missed opportunities for the local health service to learn from its errors as have other healthcare systems [6].

\section{Acknowledgements}

Many thanks to Dr Sundaresan Maiyalagan for obtaining the folinic acid

\section{References}

1. https://www.uptodate.com/contents/therapeutic-use-and-toxicityof-high-dose-methotrexate\#H1350729

2. Walling J. From Methotrexate to pemetrexed and beyond. A review of the Pharmacodynamic and clinical properties of Antifolates. Invest New Drugs. 2006;24(1):37-77.

3. Flombaum CD, Meyers PA. High-dose leucovorin as sole therapy for Methotrexate toxicity. J Clin Oncol. 1999;17:1589-1594.

4. Widemann BC, Balis FM, Kim A, Boron M, Jayaprakash N, Shalabi $A$, et al. Glucarpidase, leucovorin, and thymidine for high-dose Methotrexate-induced renal dysfunction: clinical and pharmacologic factors affecting outcome. J Clin Oncol. 2010;28(25):3979-3986. doi: 10.1200/JCO.2009.25.4540

5. http://www.gmc-uk.org/guidance/good_medical_practice/duties_ of_a_doctor.asp

6. Monisha Gupta, Marius Rademaker, John R Sullivan, et al. A decade of Australian methotrexate dosing errors. Med J Aust. 2016;205(10):485 